\title{
The Pressure Problem in the Stochastic Vortex Blob Method
}

\author{
Andrzej Nowakowski, Jacek Rokicki and Andrzej Styczek \\ Institute of Aeronautics and Applied Mechanics \\ Warsaw University of Technology \\ Nowowiejska 22/24, 00-665 Warsaw, Poland
}

\begin{abstract}
The paper deals with the problem of recovering pressure from the given vorticity and velocity fields. Both fields result from the stochastic vortex-blob simulation of the viscous incompressible flow. The vorticity field is a discontinuous function. The method is presented here to find pressure as a solution to the variational problem which forms the weak version of the momentum equation. No explicit boundary condition for pressure are required and no derivatives of vorticity have to be calculated in this approach.
\end{abstract}

\section{Introduction}

From the computational point of view the pressure field forms a free parameter in momentum equations. This free parameter is chosen in such a way that the continuity equation can be satisfied. The introduction of vorticity and the alternative formulations of the Navier-Stokes (i.e. streamfunction-vorticity or velocity-vorticity) allow to decouple purely kinematical problem from the pressure problem. In this way it is possible, as it is often claimed, to obtain the vorticity and the velocity fields prior to any pressure calculations. The pressure field is then recovered by solving suitable Poisson equation. However the choice of boundary conditions is a controversial point since no a priori boundary conditions can be prescribed (at least at solid walls). Moreover the use of Neumann boundary conditions leads to a numerically ill-posed problem. A large number of publications are devoted to identify the proper approach $[4,5,7,8]$ and to generate such boundary conditions from the momentum equation.

The other alternative is to use the variational approach $[6,9]$. which has several advantages over the approach to solve the Poisson equation directly. In the variational approach (i) no boundary conditions are needed, (ii) the ill-posedness can be addressed directly and (iii) no derivatives of vorticity have to be calculated.

The last property is especially attractive if the pressure is to be calculated from the vorticity field obtained by the stochastic vortex-blob method. In this case the vorticity field consists of a finite number of vorticity carriers (vortex-blobs) which move with the liquid and perform additional random walk to simulate viscous diffusion. The resulting global vorticity field is piecewise constant. The classical formulation of the pressure problem (via Poisson equation) will fail here since it requires calculating first derivatives of vorticity (or second derivatives of velocity).

The algorithm presented here takes full advantage of the variational approach and allows (as it is shown numerically) to obtain continuous pressure field from the discontinuous vorticity field. This variational problem is equivalent to the weak form of the Poisson equation with Neumann boundary conditions. 


\section{Basic equations}

The Navier-Stokes equations written in primitive variables for incompressible fluid are

$$
\begin{gathered}
\frac{\partial \mathbf{u}}{\partial t}+(\mathbf{u} \cdot \nabla) \mathbf{u}=-\frac{1}{\rho} \operatorname{grad} p+\nu \Delta \mathbf{u}+\mathbf{f}_{e} \\
\operatorname{div} \mathbf{u}=0
\end{gathered}
$$

where $\mathbf{u}, p, \rho$ stand for velocity vector, pressure and density $(\rho=1)$. The kinematic viscosity coefficient is denoted by $\nu$ and $\mathbf{f}_{e}$ represents the external body force field. In the above we regard the velocity and its time derivative as known everywhere (the pressure field remains unknown). We can reduce (1) to a simple abstract problem [9]

$$
\operatorname{grad} q=\mathbf{F}
$$

where

$$
\begin{aligned}
q & =\frac{u^{2}}{2}+p \quad u^{2}=\mathbf{u} \cdot \mathbf{u} \\
\mathbf{F} & =-\frac{\partial \mathbf{u}}{\partial t}+\mathbf{u} \times \omega-\nu \operatorname{curl} \omega+\mathbf{f}_{e}
\end{aligned}
$$

( $q$ may be interpreted as the total pressure). If $q$ is found by solving (3) the pressure field can be calculated from the formula $p=q-\frac{u^{2}}{2}$. However the solution does not always exist (for arbitrary F). The necessary condition for the solution to exist is

$$
\operatorname{curl} \mathbf{F}=0
$$

(it is obtained by applying curl to the both sides of (3)). Substituting the expression (5) into (6) one obtains the particular form of the necessary condition

$$
\frac{\partial \omega}{\partial t}-\operatorname{curl}(\mathbf{u} \times \omega)-\nu \triangle \omega=\operatorname{curl} \mathbf{f}_{e} \quad(\omega=\operatorname{curl} \mathbf{u})
$$

which in $2 \mathrm{D}$ case reduces to the well known scalar Helmholtz equation:

$$
\frac{\partial \zeta}{\partial t}+\mathbf{u} \nabla \zeta-\nu \triangle \zeta=\operatorname{curl} \mathbf{f}_{e}
$$

The last equation is directly simulated by the vortex-blob method and as a result (8) is satisfied by the obtained vorticity and velocity fields. This assures that ideally $\mathbf{F}$ calculated from (5) will fulfill the condition (6). In reality however the numerical representation of $\mathbf{F}$ may violate this condition and as a consequence this problem remains ill posed. Moreover the vorticity $\zeta$ is in our case not smooth enough to allow for the proper evaluation of $\mathbf{F}$. It is expected that the variational approach will help to overcome this difficulties.

\section{The existence of solution in multiconnected domains}

It was shown in the previous section that (6) forms the necessary condition for the existence of solution of the pressure problem (3). This is however not a sufficient condition if the domain is multiconnected. 
To verify this consider $x$-periodic rectangle $\Omega=\{(x, y): 0 \leq x \leq L, 0 \leq y \leq H\}$ which forms the simplest multiconnected domain. By the $x$-periodicity of $\Omega$ we understand that every continuous function on $\Omega$ has the property that $\Phi(0, y)=\Phi(L, y) \quad(0 \leq y \leq H)$. This $x$-periodic rectangle is topologically equivalent to all annular domains ( $x$ has the meaning of the meridional coordinates). Consider also the constant right-hand side $\mathbf{F}=[1,0]^{\mathrm{T}}$ which clearly satisfies the necessary condition (6). The direct integration of (3) results in

$$
q(x, y)=x+c
$$

which is not $x$-periodic since $q(L, y)-q(0, y)=L>0$. As a consequence the pressure problem has no solution in the class of continuous functions. The discontinuous pressure has in this context no physical meaning (occasionally the pressure jump may be required and prescribed).

Consider now the general case of K-connected domain $\Omega$ (see Fig. 1). The velocity and the vorticity fields are given everywhere on $\Omega$ and the resulting $\mathbf{F}$ satisfies condition (6).

If the value of $q$ is known at one point $A$ in $\Omega$ its value at the point $B$ can be calculated by integrating $\mathbf{F}$ along a line connecting $A$ and $B$

$$
q_{B}=q_{A}+\int_{A B} \mathbf{F} \cdot d \sigma
$$

and this value does not depend on the path of integration. In particular the integral above should vanish if it is taken along the contour $\gamma_{k}$

$$
[q]_{k}=\int_{\gamma_{k}} \mathbf{F} \cdot d \sigma=0
$$

otherwise $q$ would be multivalent, i.e. could be assigned more than one value at each point. The value of the jump $[q]_{k}$ (if it does not vanish) can be obtain by integration along any line surrounding $\Gamma_{k}$ (and not surrounding any other part of the boundary). In the particular case of the incompressible 2D flow problem $(4-5)$ the right hand side of the additional condition (9) can be expressed as:

$$
[q]_{k}=\int_{0}^{\Sigma_{k}} \mathbf{F} \cdot \tau d \sigma=\int_{0}^{\Sigma_{k}}-\frac{\partial u_{\tau}}{\partial t}+\zeta u_{n}-\nu \frac{\partial \zeta}{\partial n}+f_{e \tau} d \sigma
$$

where $\Sigma_{k}$ stands for the length of $\gamma_{k}$ and $\tau, \mathbf{n}$ denote respectively the tangent and the normal versors to $\gamma_{k}$. If instead of the normal and the tangential velocity components the streamfunction $\psi$ is used we obtain

$$
[q]_{k}=-\int_{0}^{\Sigma_{k}} \zeta \frac{\partial \psi}{\partial \tau}+\frac{\partial}{\partial n}\left[\nu \zeta+\frac{\partial \psi}{\partial t}\right]+f_{e \tau} d \sigma
$$

This formula when written on the fixed boundaries (with $f_{e \tau} \equiv 0$ ) results in

$$
[q]_{k}=-\nu \int_{0}^{\Sigma_{k}} \frac{\partial \zeta}{\partial n} d \sigma
$$

The corresponding no-jump condition can be interpreted as requirement that the net flux of vorticity vanishes at every disjoint part of the boundary.

However neither of (10-12) can be used in the case of piecewise constant vorticity field since then $\partial \zeta / \partial n$ is not well defined. To avoid this difficulty consider now an arbitrary annular domain $\tilde{\Omega}_{k}$ surrounding $\Gamma_{k}$ but not surrounding any other $\Gamma_{1}, \ldots, \Gamma_{K}$. Consider also the conformal transformation of $\tilde{\Omega}_{k}$ onto the periodic rectangle $\tilde{\Omega}=\{(\xi, \eta): 0 \leq \xi \leq 2 \pi, 0 \leq \eta \leq H\}$. Such 
transformation always exists. In the new coordinate system $(\xi, \eta)$ the expression $(11)$ takes the following form

$$
[q]_{k}=-\int_{0}^{2 \pi} \zeta \frac{\partial \psi}{\partial \xi}+\frac{\partial}{\partial \eta}\left[\nu \zeta+\frac{\partial \psi}{\partial t}\right] d \xi
$$

where it is assumed now that the subintegral function is taken at some $\eta=\eta_{0}=$ const and $f_{e \tau} \equiv 0$. The result does not depend on the choice of $\eta_{0}$. In particular the integration of (13) along $\eta$ will result in

$$
\begin{aligned}
H \cdot[q]_{k}= & -\int_{0}^{H} \int_{0}^{2 \pi} \zeta \frac{\partial \psi}{\partial \xi} d \xi d \eta-\nu \int_{0}^{2 \pi} \zeta(H, \xi)-\zeta(0, \xi) d \xi \\
& -\frac{\partial}{\partial t} \int_{0}^{2 \pi} \psi(H, \xi)-\psi(0, \xi) d \xi .
\end{aligned}
$$

The expression above can be calculated even for the piecewise constant vorticity distribution. It must be stressed here that the conditions (10-15) have to be implemented (as additional constraint) in the solution method that delivers velocity and vorticity fields. Without this constraint the velocity and the vorticity fields satisfying the Helmholtz equation do not represent the solution of the Navier-Stokes equation and the physically meaningful pressure field cannot be recovered. In other words the decoupling of the kinematic and the pressure problems is not complete in the case of multiconnected domains.

\section{Variational formulation}

We will assume now that the flow domain (computational domain) $\Omega \subset \mathbb{R}^{N}$ is open, bounded and connected; its boundary $\Gamma$ is at least Lipschitz continuous [5]. The usual notation of Hilbert spaces is adopted (i.e. $L^{2}(\Omega)$ denotes the space of square integrable functions on $\Omega$ and $H^{1}(\Omega)$ the space of functions with square integrable gradients).

The fundamental result concerning the orthogonal decomposition of a vector field from the space $\left[L^{2}(\Omega)\right]^{2}[5]$ is now recalled $\left(\Omega \subset \mathbb{R}^{2}\right)$

Theorem 1. For each vector function $\mathbf{F} \in\left[L^{2}(\Omega)\right]^{2}$ there exist scalar functions $q, \Phi \in H^{1}(\Omega)$ such that

$$
\begin{array}{r}
\mathbf{F}=\operatorname{grad} q+\operatorname{curl} \Phi \\
\gamma_{n}(\mathbf{F}-\operatorname{grad} q)=0
\end{array}
$$

$\left(\gamma_{n} \mathbf{v}\right.$ has the meaning of the generalized normal component of $\mathbf{v}$ on $\left.\Gamma\right)$. Moreover $\operatorname{grad} q$ and $\operatorname{curl} \Phi$ are orthogonal in $H^{1}(\Omega)$. The scalar field $q$ can be found as a solution of the following variational problem:

$$
\int_{\Omega} \nabla q \cdot \nabla \mu d \Omega=\int_{\Omega} \mathbf{F} \cdot \nabla \mu d \Omega \quad \forall \mu \in H^{1}(\Omega) .
$$

The variational problem in concern has [5] a unique solution in $H^{1}(\Omega) / \mathbb{R}$. (i.e the solution is unique within an additive constant). This solution may be regarded as a least square solution of the ill posed problem (3) in case that $\operatorname{curl} \mathbf{F} \neq 0$.

We restrict our consideration to the $2 \mathrm{D}$ hydrodynamic problem (3-5). The right hand side of (16) reduces to the sum of the four integrals $(\omega=[0,0, \zeta])$ 


$$
\begin{aligned}
I_{1} & =-\int_{\Omega} \frac{\partial \mathbf{u}}{\partial t} \cdot \nabla \mu d \Omega=-\int_{\Gamma} \mu \frac{\partial \mathbf{u}}{\partial t} \cdot \mathbf{n} d \Gamma \\
I_{2} & =-\int_{\Omega} \operatorname{curl} \zeta \cdot \nabla \mu d \Omega=-\int_{\Gamma} \zeta \frac{\partial \mu}{\partial \sigma} d \Gamma \\
I_{3} & =\int_{\Omega}(\mathbf{u} \times \omega) \cdot \nabla \mu d \Omega=\int_{\Omega} \zeta \cdot(\nabla \mu \times \mathbf{u}) d \Omega \\
I_{4} & =\int_{\Omega} \mathbf{f}_{e} \cdot \nabla \mu d \Omega
\end{aligned}
$$

where $\sigma$ denotes direction tangent to the boundary and $\mu$ stands for the arbitrary test function from $H^{1}(\Omega)$. In the above the boundary integrals were obtained [9] using the Green formula and the continuity equation (2). The second boundary integral for $I_{2}$ can be calculated only if $\nabla \mu$ can be assigned a boundary value.

\section{Numerical algorithm}

The variational problem (16) can be solved easily by means of finite element method using triangular elements and standard piecewise linear basis functions. No explicit boundary conditions are required for $q$, however the form of the right hand side suggests that the Neumann boundary conditions are simulated. Even if curl $\mathbf{F}$ does not vanish (due to the numerical reasons) the solution of the equation (16) exists. Such a solution may be regarded as a generalized solution of the original ill-posed problem (3) - the variational formulation filters out the spurious component of the right hand side.

Consider now the finite element discretization of (16) with $T_{h}$ denoting a suitable triangulation of the original region $\Omega$ ( $h$ - stands for characteristic size of the discretization). The piecewise linear approximation of the fields $\mathbf{u}$ and $q$ is denoted by $\mathbf{u}_{h}$ and $q_{h}$. The scalar vorticity field $\zeta$ is not approximated in this manner but remains in its original form. Both $\mathbf{u}_{h}$ and $\zeta$ are regarded as known and each piecewise linear test function $\mu_{h}$ has the property that it vanishes at all nodes of triangular grid except for one point. All linear combinations of all such test functions form a finite dimensional vector space $V_{h}$.

The following finite dimensional linear problem is obtained

$$
\begin{aligned}
\int_{\Omega} \nabla q_{h} \cdot \nabla \mu_{h} d \Omega & =-\int_{\Gamma} \mu_{h} \frac{\partial \mathbf{u}_{h}}{\partial t} \cdot \mathbf{n} d \Gamma+\nu \int_{\Gamma} \zeta \frac{\partial \mu_{h}}{\partial \sigma} d \Gamma+ \\
& +\int_{\Omega} \zeta \cdot\left(\nabla \mu_{h} \times \mathbf{u}_{h}\right) d \Omega+\int_{\Omega} \mathbf{f}_{e} \cdot \nabla \mu_{h} d \Omega \quad \forall \mu_{h} \in V_{h} .
\end{aligned}
$$

In the above, the time derivative is approximated by the central finite-difference formula using the information from the previous and the next time steps.

As it was mentioned earlier the vorticity field consists of a finite (large) number of blobs, each blob with constant vorticity distributed over the circle of typical diameter $D$. The global vorticity field is a piecewise constant function on $\Omega$ and the assumption that $\zeta \in H^{1}(\Omega)$ does not hold (this property is necessary to evaluate $I_{2}$ or its boundary equivalent). To avoid this difficulty one may use blobs with continuous vorticity distribution.

However, we are not interested here in the local variation of pressure in the scale of a single blob, but rather in the global averaged behaviour of pressure on a coarser grid. Indeed in the vortex-blob method the behaviour of the flowfield in the microscale has no physical meaning (no matter how 
smooth is the vorticity distribution of the single blob). In particular the averaged information should be sufficient to evaluate the resulting integral parameters (the forces and the moments). In other words the blob diameter $D$ must remain smaller than the finite-element discretization parameter $h$ (as $h \rightarrow 0$ ). In this case we can calculate all integrals in (21) using piecewise continuous vorticity distribution $\zeta$. The somewhat ambiguous boundary derivative $\partial \mu_{h} / \partial \sigma$ has in this case clear meaning since $\mu_{h}$ is a piecewise linear function on the boundary. We do not know of a strict justification of such procedure, nevertheless it may be interpreted as local averaging of discontinuous vorticity field in order to obtain smoother function on a coarser grid.

\section{Numerical results}

The test case consists of the $2 \mathrm{D}$ flow about the circular cylinder of radius $R=1$. The speed at infinity (dependent on time) is denoted by $V_{\infty}(t)$ and is given by the formula

$$
V_{\infty}(t)= \begin{cases}0 & t<0 \\ t / t_{0} & 0 \leq t \leq t_{0} \\ 1 & t>t_{0}\end{cases}
$$

The vorticity and the velocity fields, in the consecutive moments of times, are obtained by the vortex-blob method similar to the original method of Chorin [3]. The main features of this method are:

- the vortex blobs have circular support and constant vorticity,

- the viscous diffusion is simulated in whole flowfield through the random walk,

- no vortex sheets are employed

- at each time step new generation of vortex blobs is born at the solid walls,

- the circulation of each new blob at the solid boundary is found by solving the linear integral equation which guarantees that both components of velocity vanish at the boundary.

The last feature was introduced by Styczek [10] and Błażewicz and Styczek [2]. It was shown by Szumbarski and Wald [12] that the method employed here guarantees that the pressure field obtained from the vorticity and the velocity fields remains single-valued (provided the pressure is single-valued at the initial moment).

The vorticity and velocity fields used by the pressure program come from the vortex-blob program written by Styczek and Wald [11].

In our calculations the Reynolds number of the flow was based on diameter of the cylinder

$$
R e=\frac{V_{\infty} 2 R}{\nu}=\frac{2}{\nu} \quad\left(V_{\infty}=1, R=1\right)
$$

The characteristic diameter of the single vortex-blob was $\delta=0.034$. The finite element grid consisted of $160 \times 50 \times 2$ triangles. The grid had a rectangular topology.

The advantage of the used vortex method is demonstrated as it is able to capture the wide range of Reynolds numbers. In Fig. 2 we present a comparison of the calculated drag coefficient with the results of experiment [1]. In order to obtain $c_{D}$ versus $R e$ plot a number of simulations were carried out. The value of $c_{D}$ was evaluated by averaging in time. These results were obtained for developed flow with the separated vortex structure in the rear part of the cylinder. Fig. 3 shows 
the time development of the drag and lift coefficients for the flow with $R e=186000$. The numerical simulation was performed with the time step $\Delta=0.2$.

The evaluation of the pressure distribution over the surface of cylinder was also carried out. Fig. 4 shows the comparison of pressure distribution based on experimental measurements for $R e=186000$ with the calculated results. The plot presents pressure coefficient averaged around maximum lift and pressure coefficient fully averaged through the whole period. There is a general agreement with the experimental data with the exception of the separation region where calculated pressure was higher than the one obtained from experiment.

We present also the time development of the pressure field in the consecutive time steps (Figs. $5 \mathrm{a}-\mathrm{f})$. The evolution is shown staring at the nondimensional time $t U_{\infty} / R=100$ of the simulation when the flow is fully developed. The reader may note that the zero lift case corresponds to an almost symmetric wake in an immediate neighborhood of the cylinder (Fig. 5e).

\section{Acknowledgments}

This work has been supported by the KBN (Polish State Committee for Scientific Research) Grant 3-3314-92-03. We are also indebted to Professor Yves Gagnon, from the Université de Moncton, Canada for encouraging us to proceed with the research on the pressure problem.

\section{References}

[1] Anderson, J.D., Fundamentals of Aerodynamics, McGraw-Hill, New York, 1991.

[2] Błażewicz, J. and Styczek, A., "The Stochastic Simulation of the Viscous Fluid Flow Past an Airfoil," J. Theo. and Appl. Mech., 31 (1, 4), 1993.

[3] Chorin, A.J., "Numerical Study of Slightly Viscous Flow," J. Fluid Mech., 57, pp. 785-796, 1973.

[4] Dennis, S.C.R. and Quartapelle, L., "Some Uses of Green's Theorem in Solving the NavierStokes Equations," Int. J. Num. Meth. Fluids, 9, pp. 871-890, 1989.

[5] Girault, V. and Raviart, P.-A., Finite Element Approximation of the Navier-Stokes Equations, Springer-Verlag, Heidelberg, 1981.

[6] Gunzburger, M.O., Finite Element Methods for Viscous Incompressible Flows, Academic Press, San Diego, 1989.

[7] Quartapelle, L. and Napolitano, M., "Integral Conditions for Pressure in the Computation of Incompressible Viscous Flows," J. Comp. Phys., 62, pp. 340-348, 1986.

[8] Rokicki, J. and Floryan, J.M., "Domain Decomposition and the Compact Fourth-Order Algorithm for the Navier-Stokes Equations," J. Comp. Phys., 116, pp. 79-96, 1995.

[9] Rokicki, J. and Styczek, A., "How to Calculate Pressure out of Velocity and Vorticity Fields," Arch. Mech. Eng., XXXIX (Z4), pp. 351-355, 1992.

[10] Styczek, A., "The Vortex-Blob Method of Simulating the Viscous Liquid Motion," Arch. Mech. Eng., XXXIV, pp. 225-241, 1987. 
[11] Styczek, A. and Wald, P., "The Fast and Efficient Vortex-Blob Simulation of the Flow Past the Circular Cylinder," Arch. Mech. Eng., XLII, pp. 281-298, 1995.

[12] Szumbarski, J. and Wald, P., "The Stochastic Vortex Sumulation of an Unsteady Viscous Flow in a Multiconnected Domain," Proc. Sec. Int'l. Workshop on Vortex Flows and Related Numerical Methods, Montreal, Canada, pp. 125-126, 1995.

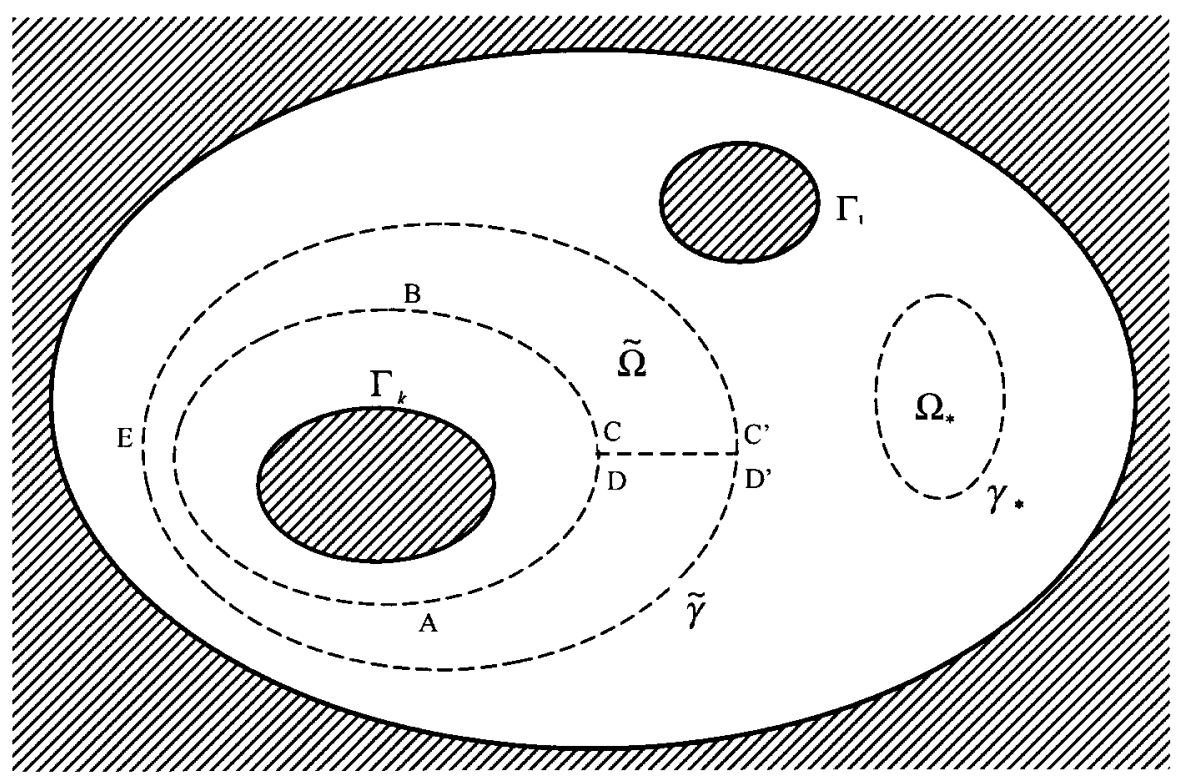

Figure 1: Typical multiconnected domain.

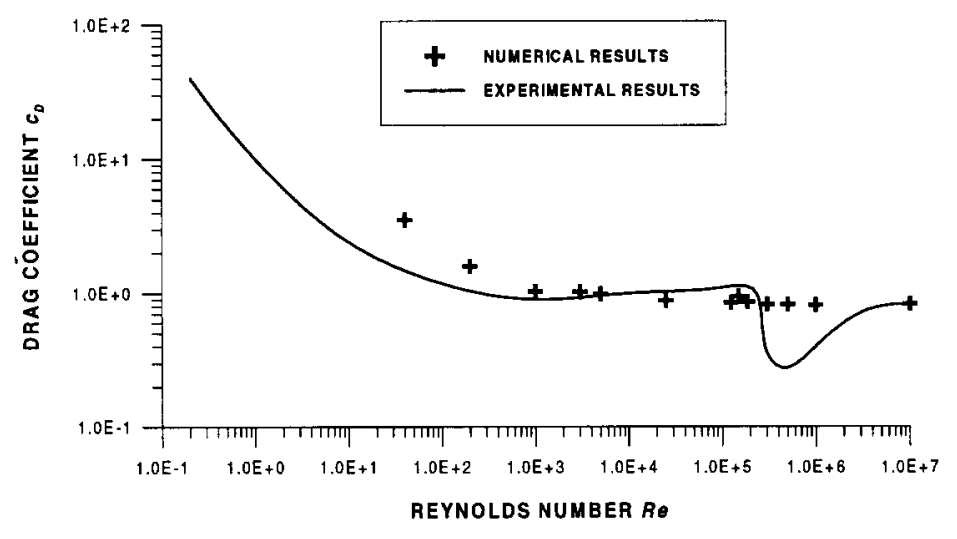

Figure 2: Comparison of the calculated drag coefficient $c_{D}$ with the experimental results [1]. 


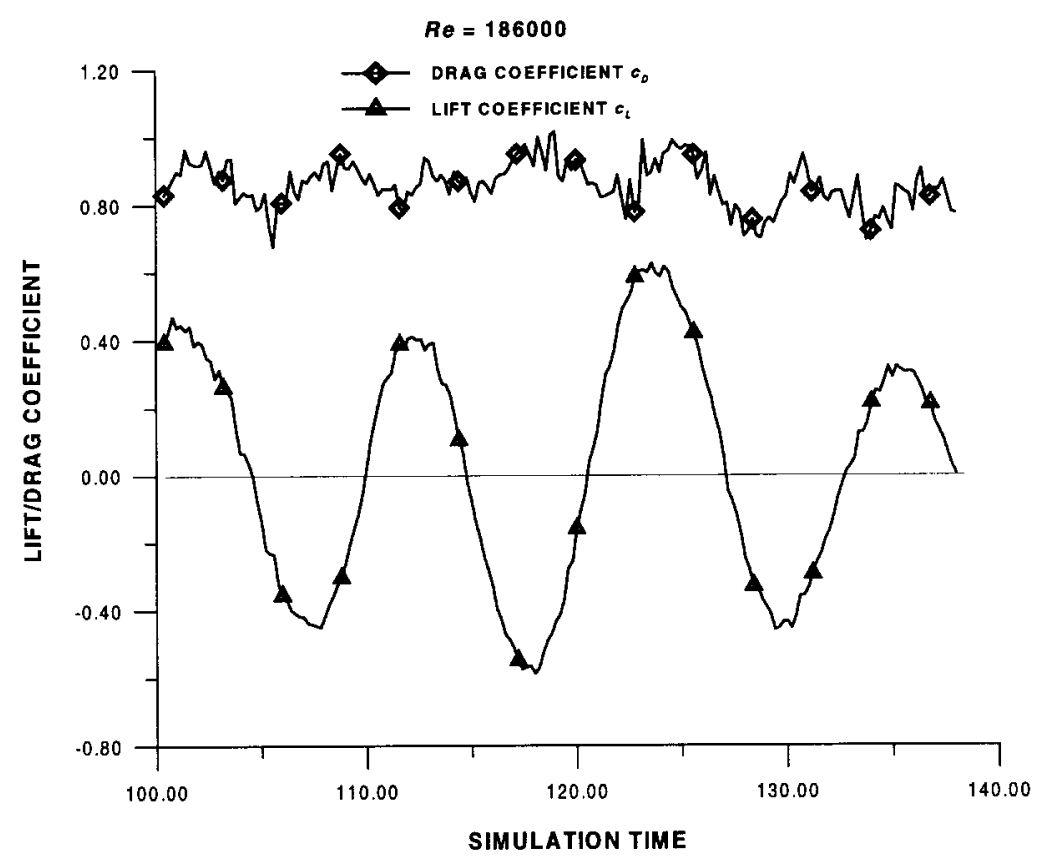

Figure 3: Time history of drag coefficient $c_{D}$ and lift coefficient $c_{L}$ for $R e=186000$.

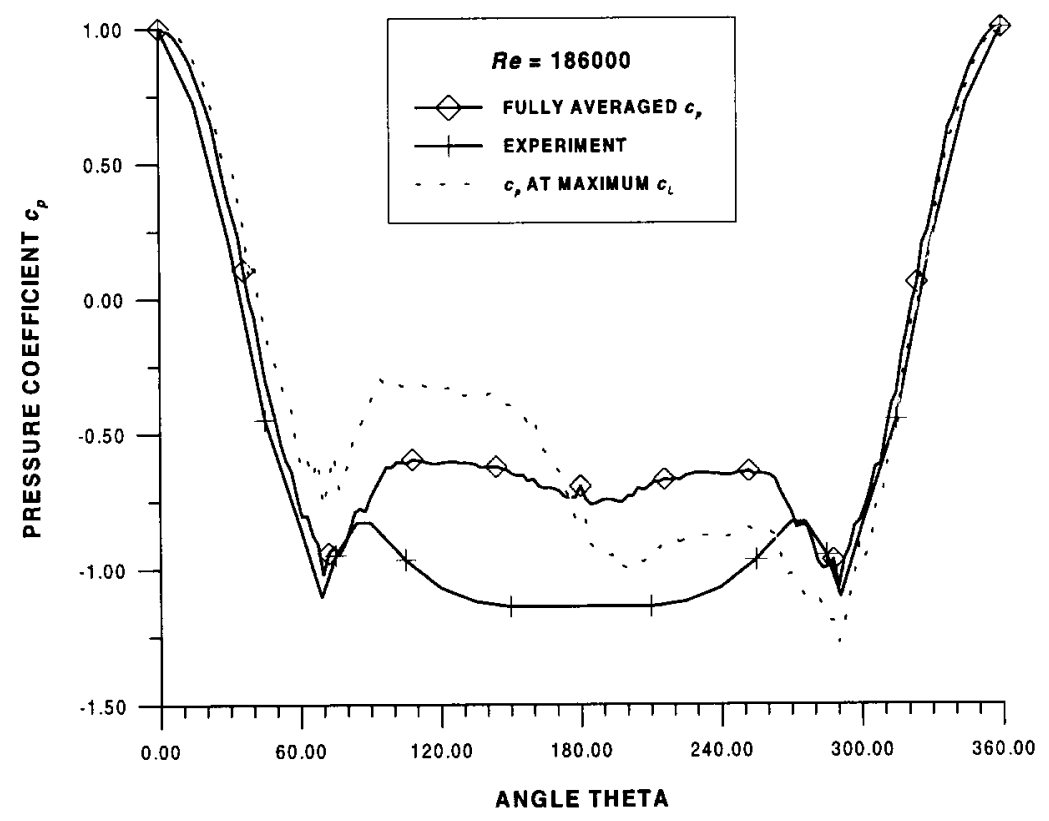

Figure 4: Pressure distribution over a circular cylinder for $R e=186000$, comparison with the experimental results [1]. 


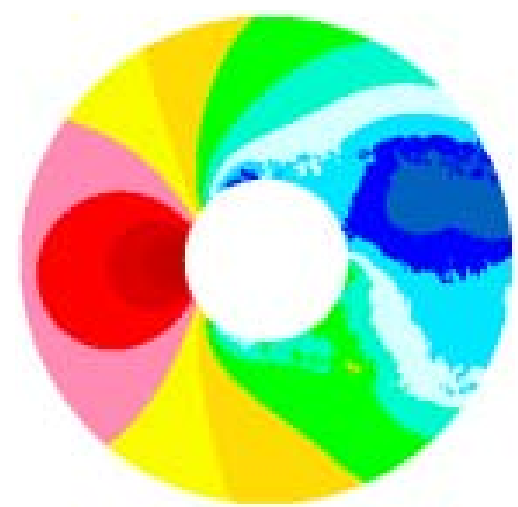

Fig.5a $t=112$

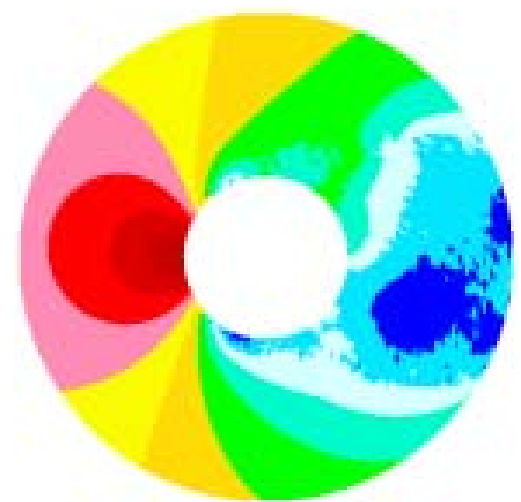

Fig.5c $t=116$

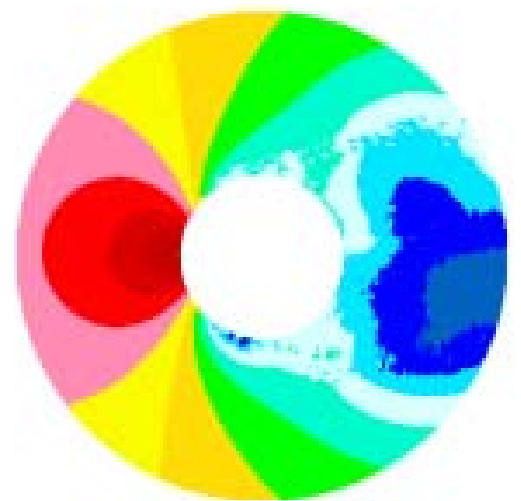

Fig.5e $t=120$

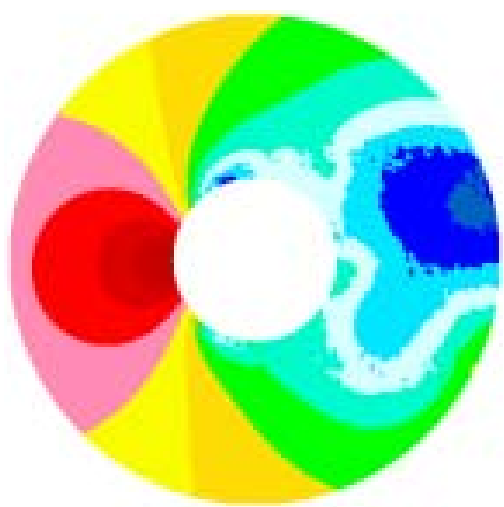

Fig. $5 \mathrm{~b} t=114$

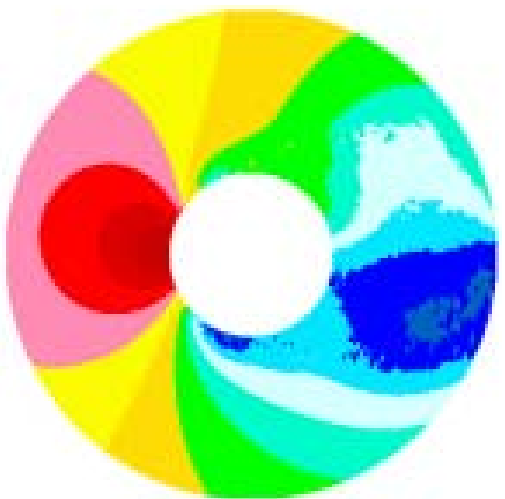

Fig. $5 \mathrm{~d} t=118$

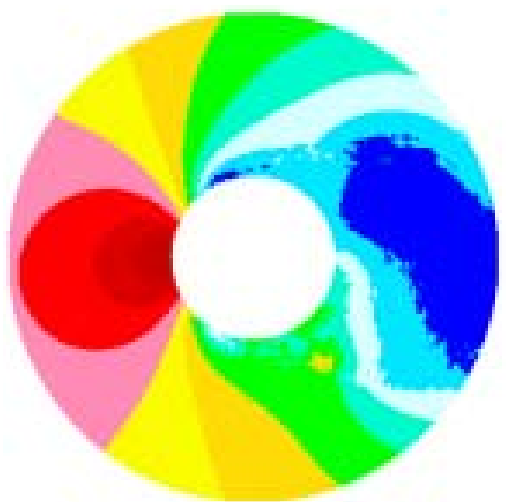

Fig.5f $t=122$
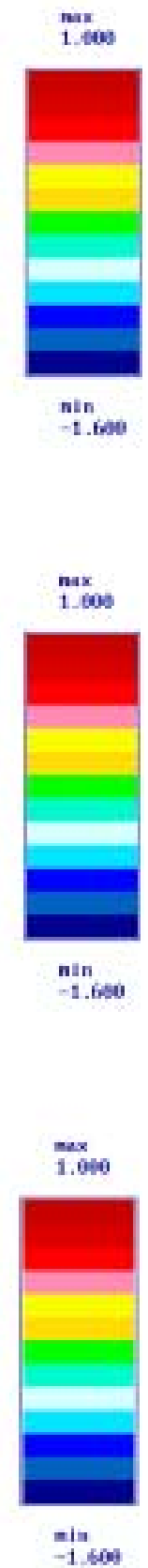

Figure 5: The pressure coefficient $c_{p}$ at the nondimensional time $t, R e=186000$. The inner boundary corresponds to the cylinder surface, the outer boundary is fictitious. 